\title{
A Proof of the Identifiability of a Subspace-Based Blind Channel Estimation for OFDM Systems
}

\author{
Yonghong Zeng, Member, IEEE, and Tung Sang Ng, Fellow, IEEE
}

\begin{abstract}
Muquet et al. have proposed a subspace-based blind method for estimating the channel responses of cyclic-prefixed OFDM systems. Their proof for the channel identifiability uses the roots of channel transfer function and therefore depends on the channel order. When only an upper bound is known for the channel order, the proof fails. In this letter, a new proof for the identifiability is given which does not require knowledge of the precise channel order and can handle various choices of length of the OFDM block.
\end{abstract}

Index Terms-Blind channel identification, cyclic prefix, identifiability, orthogonal frequency division multiplexing (OFDM), subspace method.

\section{INTRODUCTION}

$\mathbf{O}$ RTHOGONAL frequency division multiplexing (OFDM) is a special case of multicarrier modulation, which can effectively mitigate the effects of multipath propagation and hence increase data rate [1]. Also, OFDM system can be equalized in frequency domain by using the fast Fourier transform (FFT), which is fast and efficient. Subspace-based method is a major approach for blind channel estimation in wireless communication [2]-[5]. The basic idea for any subspace-based method is the same, that is, using the obtained signal or noise subspace (from the statistical information of output signals) to identify the channels. However, channel identifiability and its proof vary greatly for different systems. In [6], Muquet et al. have proposed a subspace-based blind method for estimating the channel responses of OFDM systems. In the proof [6, Appendix B] for the channel identifiability, they assume that the channel transfer function has $L$ zeros (distinct or multiple), and these $L$ zeros are used to construct a matrix $\mathbf{V}$ (see [6]) of size $L \times L$, where $L$ is an upper bound of the channel order. If $L$ is not the real channel order (this is the case for most practical applications), that is, the real order is less than $L$, it is obvious that the channel transfer function surely has less than $L$ zeros. Therefore, the matrix $\mathbf{V}$ cannot be constructed and the proof fails. In this letter, a new proof for the identifiability is given which does not use the precise channel order and can handle various choices of length of the OFDM block. Also, it is shown that, in most cases, even if channel zeros hit some of the OFDM subcarriers, the channel

Manuscript received September 4, 2003; revised February 17, 2004. This work was supported by the Research Grants Council of the Hong Kong SAR, China, under Grant HKU 7164/04E. The associate editor coordinating the review of this manuscript and approving it for publication was Dr. Mounir Ghogho.

The authors are with the Department of Electrical and Electronic Engineering, The University of Hong Kong, Hong Kong (e-mail: yhzeng@eee.hku.hk; tsng@eee.hku.hk).

Digital Object Identifier 10.1109/LSP.2004.833584 is still identifiable. The concrete steps for finding the channel in [6] remains valid and therefore is not discussed here.

\section{SYSTEM DESCRIPTION}

The system model considered here is the same as that in [6]. Let $L$ be the length of the cyclic prefix (CP) and also an upper bound of the channel order. The channel response is denoted by $h(l)(l=0,1, \ldots, L)$. We can always assume that $h(0) \neq 0$ (otherwise the zeros preceding the first nonzero coefficient can be removed). It is assumed that each OFDM block consists of $M=\mu L$ symbols ([6] only considers the case of $\mu=4$ ), where $\mu$ is an integer. Let $\mathbf{s}_{\mathrm{cp}}(k)$ be the OFDM block at time epoch $k$ after implementing the inverse FFT and cyclic prefixing. Then, the number of symbols in such a block is $P=(\mu+1) L$. Let $\mathbf{r}_{\mathrm{cp}}(k)$ and $\mathbf{n}(k)$ be the received block and ambient noise, respectively. All blocks can be split into $\mu+1$ subblocks of length $L$

$$
\begin{aligned}
\mathbf{r}_{\mathrm{cp}}(k) & =\left[\mathbf{r}_{0}(k)^{T}, \ldots, \mathbf{r}_{\mu}(k)^{T}\right]^{T} \\
\mathbf{s}_{\mathrm{cp}}(k) & =\left[\mathbf{s}_{0}(k)^{T}, \ldots, \mathbf{s}_{\mu}(k)^{T}\right]^{T} \\
\mathbf{n}(k) & =\left[\mathbf{n}_{0}(k)^{T}, \ldots, \mathbf{n}_{\mu}(k)^{T}\right]^{T}
\end{aligned}
$$

where $\mathbf{s}_{0}(k)$ is the cyclic prefix, that is, $\mathbf{s}_{0}(k)=\mathbf{s}_{\mu}(k)$. Let us consider two consecutive blocks and define

$\begin{aligned} \overline{\mathbf{r}}(k) & =\left[\mathbf{r}_{1}(k-1)^{T}, \ldots, \mathbf{r}_{\mu}(k-1)^{T}, \mathbf{r}_{0}(k)^{T}, \ldots, \mathbf{r}_{\mu}(k)^{T}\right]^{T} \\ \overline{\mathbf{s}}(k) & =\left[\mathbf{s}_{1}(k-1)^{T}, \ldots, \mathbf{s}_{\mu}(k-1)^{T}, \mathbf{s}_{1}(k)^{T}, \ldots, \mathbf{s}_{\mu}(k)^{T}\right]^{T} \\ \overline{\mathbf{n}}(k) & =\left[\mathbf{n}_{1}(k-1)^{T}, \ldots, \mathbf{n}_{\mu}(k-1)^{T}, \mathbf{n}_{0}(k)^{T}, \ldots, \mathbf{n}_{\mu}(k)^{T}\right]^{T} .\end{aligned}$

If we use $\mathbf{C}_{0}=\mathbf{C}_{0}(h)$ to denote the $L \times L$ lower triangular Toeplitz matrix with first column $[h(0), \ldots, h(L-1)]^{T}$ and first row $[h(0), 0, \ldots, 0]$, and $\mathbf{C}_{1}=\mathbf{C}_{1}(h)$ to denote the $L \times L$ upper triangular Toeplitz matrix with first column $[h(L), 0, \ldots, 0]^{T}$ and first row $[h(L), \ldots, h(1)]$, it is easy to show that

$$
\overline{\mathbf{r}}(k)=\mathbf{H}(h) \overline{\mathbf{s}}(k)+\overline{\mathbf{n}}(k)
$$

where $\mathbf{H}(h)$ is a $(2 M+L) \times 2 M$ matrix defined as follows: if $\mu=1$,

$$
\mathbf{H}(h)=\left[\begin{array}{cc}
\mathbf{C}_{0}+\mathbf{C}_{1} & 0 \\
\mathbf{C}_{1} & \mathbf{C}_{0} \\
0 & \mathbf{C}_{0}+\mathbf{C}_{1}
\end{array}\right]
$$

if $\mu \geq 2$

$$
\mathbf{H}(h)=\left[\begin{array}{ll}
\mathbf{A} & 0 \\
\alpha & \beta \\
0 & \mathbf{A}
\end{array}\right]
$$


where $\mathbf{A}$ is an $M \times M$ matrix defined as

$$
\mathbf{A}=\left[\begin{array}{ccccc}
\mathbf{C}_{0} & & & & \mathbf{C}_{1} \\
\mathbf{C}_{1} & \mathbf{C}_{0} & & & \\
& \ddots & \ddots & & \\
& & \ddots & \ddots & \\
& & & \mathbf{C}_{1} & \mathbf{C}_{0}
\end{array}\right]
$$

where $\alpha$ and $\beta$ are two $L \times M$ matrices as

$$
\alpha=\left[\begin{array}{llll}
0 & \cdots & 0 & \mathbf{C}_{1}
\end{array}\right], \quad \beta=\left[\begin{array}{llll}
0 & \cdots & 0 & \mathbf{C}_{0}
\end{array}\right] .
$$

Please note that $\mathbf{C}_{0}$ is invertible since $h(0) \neq 0$. For $\mu \geq 2$, it is easy to show that after some elementary column transform, the matrix $\mathbf{H}(h)$ can be turned into $\mathbf{H}^{\prime}(h)$ as

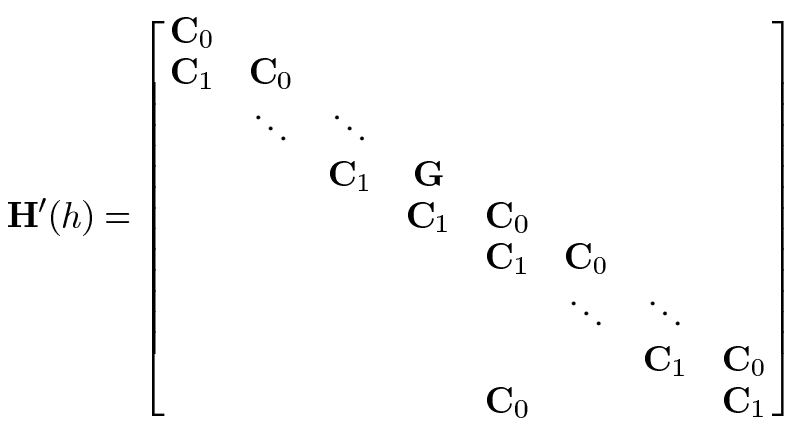

where

$$
\mathbf{G}=\mathbf{C}_{0}+(-1)^{\mu-1}\left(\mathbf{C}_{1} \mathbf{C}_{0}^{-1}\right)^{\mu-1} \mathbf{C}_{1}
$$

\section{PRoOF OF THE IDENTIFIABILITY}

We use $\operatorname{span}(\mathbf{H}(h))$ to denote the vector space spanned by the column vectors of $\mathbf{H}(h)$, that is, all possible linear combinations of the column vectors. $\operatorname{Span}(\mathbf{H}(h))$ is also called the signal subspace, which can be determined by eigen-decomposition of the statistical auto-correlation matrix of the received signal. Any subspace-based method can only determine this subspace. In general, knowing $\operatorname{span}(\mathbf{H}(h))$ cannot determine matrix $\mathbf{H}(h)$. However, for some matrix with special structure, it is possible to determine $\mathbf{H}(h)$ by $\operatorname{span}(\mathbf{H}(h))$ up to certain ambiguity. Based on its structure, we will prove in this section that $\mathbf{H}(h)$ is uniquely determined by $\operatorname{span}(\mathbf{H}(h))$ subject to a scalar ambiguity.

Lemma 1: Let $\mathbf{D}$ and $\mathbf{E}$ be two $J(N+I) \times K N(I \geq 0)$ matrices, respectively, defined as

$$
\mathbf{D}=\left[\begin{array}{cccc}
\mathbf{D}_{11} & & & \\
\mathbf{D}_{21} & \mathbf{D}_{22} & & \\
\vdots & \vdots & \ddots & \\
\mathbf{D}_{N 1} & \mathbf{D}_{N 2} & \cdots & \mathbf{D}_{N N} \\
\vdots & \vdots & & \vdots \\
\mathbf{D}_{(N+I) 1} & \mathbf{D}_{(N+I) 2} & \cdots & \mathbf{D}_{(N+I) N}
\end{array}\right]
$$

$$
\mathbf{E}=\left[\begin{array}{cccc}
\mathbf{E}_{11} & & & \\
\mathbf{E}_{21} & \mathbf{E}_{22} & & \\
\vdots & \vdots & \ddots & \\
\mathbf{E}_{N 1} & \mathbf{E}_{N 2} & \cdots & \mathbf{E}_{N N} \\
\vdots & \vdots & & \vdots \\
\mathbf{E}_{(N+I) 1} & \mathbf{E}_{(N+I) 2} & \cdots & \mathbf{E}_{(N+I) N}
\end{array}\right]
$$

where $\mathbf{D}_{j k}$ and $\mathbf{E}_{j k}$ are matrices of size $J \times K(J \geq K)$, and $\mathbf{D}_{k k}$ and $\mathbf{E}_{k k}$ are of full column rank. Then, if $\operatorname{span}(\mathbf{D})=$ $\operatorname{span}(\mathbf{E})$, there exists a block lower triangular $K N \times K N$ invertible matrix $\mathbf{F}$ defined as

$$
\mathbf{F}=\left[\begin{array}{cccc}
\mathbf{F}_{11} & & & \\
\mathbf{F}_{21} & \mathbf{F}_{22} & & \\
\vdots & \vdots & \ddots & \\
\mathbf{F}_{N 1} & \mathbf{F}_{N 2} & \cdots & \mathbf{F}_{N N}
\end{array}\right]
$$

where $\mathbf{F}_{j k}$ are matrices of size $K \times K$ and $\mathbf{F}_{k k}$ invertible, such that

$$
\mathbf{E}=\mathbf{D F}
$$

Proof: It is easy to verify that $\operatorname{span}(\mathbf{D})=\operatorname{span}(\mathbf{E})$ if and only if there exists a $K N \times K N$ invertible matrix $\mathbf{F}$ such that

$$
\mathbf{E}=\mathbf{D F}
$$

We section $\mathbf{F}$ into blocks and express it as

$$
\mathbf{F}=\left[\begin{array}{cccc}
\mathbf{F}_{11} & \mathbf{F}_{12} & \cdots & \mathbf{F}_{1 N} \\
\mathbf{F}_{21} & \mathbf{F}_{22} & \cdots & \mathbf{F}_{2 N} \\
\vdots & \vdots & & \vdots \\
\mathbf{F}_{N 1} & \mathbf{F}_{N 2} & \cdots & \mathbf{F}_{N N}
\end{array}\right]
$$

where $\mathbf{F}_{i j}$ are $K \times K$ matrices.

Now we prove that $\mathbf{F}$ must be a block lower triangular matrix and $\mathbf{F}_{k k}$ invertible by using the mathematical induction. The $i$ th block row of $\mathbf{E}$ is the multiplication of the $i$ th block row of $\mathbf{D}$ and matrix F. Considering the first block row, we have

$$
\mathbf{D}_{11} \mathbf{F}_{11}=\mathbf{E}_{11}, \mathbf{D}_{11} \mathbf{F}_{1 j}=0, \quad j=2, \ldots, N .
$$

Since $\mathbf{D}_{11}$ and $\mathbf{E}_{11}$ are of full column rank, we know that $\mathbf{F}_{11}$ must also be of full rank, that is, $\mathbf{F}_{11}$ is invertible. Also, $\mathbf{F}_{1 j}=$ $0, j=2, \ldots, N$.

Now assuming that $\mathbf{F}_{i j}=0$ and $\mathbf{F}_{i i}$ invertible, where $i=$ $1, \ldots, k$, and $j \geq i+1$, we want to show that $\mathbf{F}_{k+1, j}=0(j \geq$ $k+2)$ and $\mathbf{F}_{k+1, k+1}$ invertible. Considering the $(k+1)$ th block row, based on the assumption we have

$$
\begin{aligned}
\mathbf{D}_{k+1, k+1} \mathbf{F}_{k+1, k+1} & =\mathbf{E}_{k+1, k+1}, \\
\mathbf{D}_{k+1, k+1} \mathbf{F}_{k+1, j} & =0, \quad j=k+2, \ldots, N .
\end{aligned}
$$

Since $\mathbf{D}_{k+1, k+1}$ and $\mathbf{E}_{k+1, k+1}$ are of full column rank, we know that $\mathbf{F}_{k+1, k+1}$ is invertible. Furthermore, the full column rank property of $\mathbf{D}_{k+1, k+1}$ means that $\mathbf{F}_{k+1, j}=0, j=k+$ 
$2, \ldots, N$. Based on the mathematical induction law, we know that $\mathbf{F}$ is a block lower triangular matrix with all its diagonal blocks invertible.

Now assume that there is another channel with response $\hat{h}(l)(l=0,1, \ldots, L)$ such that $\operatorname{span}(\mathbf{H}(h))=\operatorname{span}(\mathbf{H}(\hat{h}))$. For simplicity, let $\hat{\mathbf{C}}_{0}=\mathbf{C}_{0}(\hat{h}), \hat{\mathbf{C}}_{1}=\mathbf{C}_{1}(\hat{h})$.

Theorem 1: Assume that $h(0) \neq 0, \hat{h}(0) \neq 0$, and $\mathbf{G}=\mathbf{C}_{0}+(-1)^{\mu-1}\left(\mathbf{C}_{1} \mathbf{C}_{0}^{-1}\right)^{\mu-1} \mathbf{C}_{1}, \hat{\mathbf{G}}=\hat{\mathbf{C}}_{0}+$ $(-1)^{\mu-1}\left(\hat{\mathbf{C}}_{1} \hat{\mathbf{C}}_{0}^{-1}\right)^{\mu-1} \hat{\mathbf{C}}_{1}$ are invertible. If $\operatorname{span}(\mathbf{H}(h))=$ $\operatorname{span}(\mathbf{H}(\hat{h}))$, there exists a nonzero constant $\lambda$ such that $\hat{h}(l)=\lambda h(l), l=0,1, \ldots, L$.

Proof: We first consider the case of $\mu \geq 2 . \operatorname{span}(\mathbf{H}(h))=$ $\operatorname{span}(\mathbf{H}(\hat{h}))$ if and only if there exists a $2 M \times 2 M$ invertible matrix $\mathbf{F}$ such that $\mathbf{H}(\hat{h})=\mathbf{H}(h) \mathbf{F}$. Since $\mathbf{H}(\hat{h})$ and $\mathbf{H}(h)$ can be turned into $\mathbf{H}^{\prime}(\hat{h})$ and $\mathbf{H}^{\prime}(h)$ respectively by column elementary transform, it is obvious that there exists a $2 M \times$ $2 M$ invertible matrix $\mathbf{F}^{\prime}$ such that $\mathbf{H}^{\prime}(\hat{h})=\mathbf{H}^{\prime}(h) \mathbf{F}^{\prime}$. From the structure of $\mathbf{H}^{\prime}(\hat{h}), \mathbf{H}^{\prime}(h)$ and Lemma 1 , we know that $\mathbf{F}^{\prime}$ must be a block lower triangular matrix as

$$
\mathbf{F}^{\prime}=\left[\begin{array}{cccc}
\mathbf{F}_{11}^{\prime} & & & \\
\mathbf{F}_{21}^{\prime} & \mathbf{F}_{22}^{\prime} & & \\
\vdots & \vdots & \ddots & \\
\mathbf{F}_{2 \mu, 1}^{\prime} & \mathbf{F}_{2 \mu, 2}^{\prime} & \cdots & \mathbf{F}_{2 \mu, 2 \mu}^{\prime}
\end{array}\right]
$$

where $\mathbf{F}_{i j}^{\prime}$ are $L \times L$ matrices and $\mathbf{F}_{i i}^{\prime}$ invertible. For simplicity, we use $\mathbf{B}$ to denote the matrix $\mathbf{F}_{2 \mu, 2 \mu}^{\prime}$. Considering the last block column of $\mathbf{H}^{\prime}(\hat{h})$, we see that

$$
\left[\begin{array}{c}
0 \\
\vdots \\
0 \\
\hat{\mathbf{C}}_{0} \\
\hat{\mathbf{C}}_{1}
\end{array}\right]=\mathbf{H}^{\prime}(h)\left[\begin{array}{c}
0 \\
\vdots \\
0 \\
\mathbf{B}
\end{array}\right]=\left[\begin{array}{c}
0 \\
\vdots \\
0 \\
\mathbf{C}_{0} \mathbf{B} \\
\mathbf{C}_{1} \mathbf{B}
\end{array}\right] .
$$

So, we have $\hat{\mathbf{C}}_{0}=\mathbf{C}_{0} \mathbf{B}, \hat{\mathbf{C}}_{1}=\mathbf{C}_{1} \mathbf{B}$, that is, $\hat{\mathbf{C}}=\mathbf{C B}$, where $\mathbf{C}=\left[\begin{array}{ll}\mathbf{C}_{0}^{T} & \mathbf{C}_{1}^{T}\end{array}\right]^{T}$ and $\hat{\mathbf{C}}=\left[\begin{array}{ll}\hat{\mathbf{C}}_{0}^{T} & \hat{\mathbf{C}}_{1}^{T}\end{array}\right]^{T}$ have the forms as follows:

$$
\begin{aligned}
\mathbf{C} & =\left[\begin{array}{ccc}
h(0) & & \\
\vdots & \ddots & \\
h(L-1) & \cdots & h(0) \\
h(L) & \cdots & h(1) \\
& \ddots & \vdots \\
& & h(L)
\end{array}\right] \\
\hat{\mathbf{C}} & =\left[\begin{array}{ccc}
\hat{h}(0) & & \\
\vdots & \ddots & \\
\hat{h}(L-1) & \cdots & \hat{h}(0) \\
\hat{h}(L) & \cdots & \hat{h}(1) \\
& \ddots & \vdots \\
& & \hat{h}(L)
\end{array}\right] .
\end{aligned}
$$

Since $\mathbf{B}$ is invertible, it is obvious that $\operatorname{span}(\mathbf{C})=\operatorname{span}(\hat{\mathbf{C}})$. So, noticing the structure of $\mathbf{C}, \hat{\mathbf{C}}$ and using Lemma 1 again we know that there exists an invertible lower triangular matrix $\mathbf{W}$ such that $\hat{\mathbf{C}}=\mathbf{C W}$. Comparing the last column of $\hat{\mathbf{C}}$ and
C, we see that there is a nonzero constant $\lambda$ such that $\hat{h}(l)=$ $\lambda h(l), l=0,1, \ldots, L$.

Now we consider the case of $\mu=1$. Based on Lemma 1, we know that there is an invertible matrix $\mathbf{A}$ such that $\hat{\mathbf{C}}_{0}=$ $\mathbf{C}_{0} \mathbf{A}, \hat{\mathbf{C}}_{0}+\hat{\mathbf{C}}_{1}=\left(\mathbf{C}_{0}+\mathbf{C}_{1}\right) \mathbf{A}$. Hence we also have $\hat{\mathbf{C}}_{0}=$ $\mathbf{C}_{0} \mathbf{A}, \hat{\mathbf{C}}_{1}=\mathbf{C}_{1} \mathbf{A}$, that is, $\hat{\mathbf{C}}=\mathbf{C A}$. So, the theorem is also correct.

It is possible to extend the results above to the case of $M \neq$ $\mu L$. For example, if $M \geq 2 L$ and $M$ is not a multiple of $L$, the input/output (3) can be replaced by (refer to [6, Appendix A] for details)

$$
\overline{\mathbf{r}}(k)=\overline{\mathbf{H}}(h) \overline{\mathbf{s}}(k)+\overline{\mathbf{n}}(k)
$$

where $\overline{\mathbf{H}}(h)$ is a $(2 M+L) \times 2 M$ matrix defined as

$$
\overline{\mathbf{H}}(h)=\left[\begin{array}{cccc}
\mathbf{C}_{0}^{\prime} & \mathbf{C}_{1}^{\prime} & 0 & 0 \\
\mathbf{C}_{1}^{\prime \prime} & \mathbf{C}_{0} & 0 & 0 \\
0 & \mathbf{C}_{1} & 0 & \mathbf{C}_{0} \\
0 & 0 & \mathbf{C}_{0}^{\prime} & \mathbf{C}_{1}^{\prime} \\
0 & 0 & \mathbf{C}_{1}^{\prime \prime} & \mathbf{C}_{0}
\end{array}\right]
$$

where $\mathbf{C}_{0}$ and $\mathbf{C}_{1}$ are defined as previously, $\mathbf{C}_{0}^{\prime}$ is an $(M-L) \times(M-L)$ Toeplitz matrix with first column $[h(0), \ldots, h(L), 0, \ldots, 0]^{T}$ and first row $[h(0), 0, \ldots, 0]$, and $\mathbf{C}_{1}^{\prime}=\left[\mathbf{C}_{1}^{T}, 0\right]^{T}, \mathbf{C}_{1}^{\prime \prime}=\left[0, \mathbf{C}_{1}\right]$. After some elementary column transforms, the matrix $\overline{\mathbf{H}}(h)$ can be turned to

$$
\overline{\mathbf{H}}^{\prime}(h)=\left[\begin{array}{cccc}
\mathbf{C}_{0}^{\prime} & 0 & 0 & 0 \\
\mathbf{C}_{1}^{\prime \prime} & \mathbf{Q} & 0 & 0 \\
0 & \mathbf{C}_{1} & \mathbf{C}_{0} & 0 \\
0 & 0 & \mathbf{C}_{1}^{\prime} & \mathbf{C}_{0}^{\prime} \\
0 & 0 & \mathbf{C}_{0} & \mathbf{C}_{1}^{\prime \prime}
\end{array}\right]
$$

where $\mathbf{Q}=\mathbf{C}_{0}-\mathbf{C}_{1}^{\prime \prime}\left(\mathbf{C}_{0}^{\prime}\right)^{-1} \mathbf{C}_{1}^{\prime}$. Like $\mathbf{H}^{\prime}(h), \overline{\mathbf{H}}^{\prime}(h)$ is also a lower triangular block Toeplitz matrix. So, it is possible to generalize Lemma 1 and Theorem 1 for this case.

\section{CONDITION FOR THE IDENTIFIABILITY}

The only condition for the identifiability is $\mathbf{G}=$ $\mathbf{C}_{0}+(-1)^{\mu-1}\left(\mathbf{C}_{1} \mathbf{C}_{0}^{-1}\right)^{\mu-1} \mathbf{C}_{1}$ invertible. When $\mu=1$, the condition turns to: $\mathbf{C}_{0}+\mathbf{C}_{1}$ invertible. Since $\mathbf{C}_{0}+\mathbf{C}_{1}$ is a cyclic matrix with first row $[h(L)+h(0), h(L-1), \ldots, h(1)]$, it is invertible if and only if the length- $L$ discrete Fourier transform of the first row has no zero coefficients. This condition will be invalid if $h(L)=0$ and channel zeros meet the OFDM subcarriers (note that $M=L$ ). When $\mu \geq 2$, which is the case for most practical applications [1], the physical meaning of the condition in communication remains to be investigated. However, it seems that even if channel zeros hit some of the OFDM subcarriers, the condition is still valid.

\section{CONCLUSION}

In this letter, a new proof for the subspace-based channel identifiability of the cyclic-prefixed OFDM system has been given. The proof does not assume the precise channel order and can handle various choices of length of the OFDM block. Furthermore, it is shown that, in most cases, even if channel zeros 
hit some of the OFDM subcarriers, the channel is still identifiable.

\section{REFERENCES}

[1] J. Heiskala and J. Terry, OFDM Wireless LANs: A Theoretical and Practical Guide. Indianapolis, IN: Sams, 2002.

[2] K. Abed-Meraim, P. Loubaton, and E. Moulines, "A subspace algorithm for certain blind identification problem," IEEE Trans. Inform. Theory, vol. 32 , no. 2 , pp. 499-511, 1997.
[3] E. Moulines, P. Duhamel, J. F. Cardoso, and S. Mayrargue, "Subspace methods for the blind identification of multichannel FIR filters," IEEE Trans. Signal Processing, vol. 43, pp. 516-525, Feb. 1995.

[4] Z. Ding and Y. Li, Blind Equalization and Identification. New York: Marcel Dekker, 2001

[5] G. B. Giannakis, Y. Hua, P. Stoica, and L. Tong, Signal Processing Advances in Wireless \& Mobile Communications. Englewood Cliffs, $\mathrm{NJ}$ : Prentice-Hall, 2001, vol. 1.

[6] B. Muquet, M. D. Courville, and P. Duhamel, "Subspace-based blind and semi-blind channel estimation for OFDM systems," IEEE Trans. Signal Processing, vol. 50, pp. 1699-1712, July 2002. 